\title{
Self-consistent relativistic quasiparticle random-phase approximation and its applications to charge-exchange excitations
}

\author{
Z. M. Niu (牛中明), ${ }^{1,2}$ Y. F. Niu (牛一斐), ${ }^{3}$ H. Z. Liang (梁豪兆), ${ }^{4,5},{ }^{*}$ W. H. Long (龙文辉), ${ }^{6}$ and J. Meng (孟杰) ${ }^{7,8,9, \dagger}$ \\ ${ }^{1}$ School of Physics and Material Science, Anhui University, Hefei 230039, China \\ ${ }^{2}$ Interdisciplinary Theoretical Science Research Group, RIKEN, Wako 351-0198, Japan \\ ${ }^{3}$ ELI-NP, "Horia Hulubei” National Institute for Physics and Nuclear Engineering, \\ 30 Reactorului Street, RO-077125 Bucharest-Magurele, Romania \\ ${ }^{4}$ RIKEN Nishina Center, Wako 351-0198, Japan \\ ${ }^{5}$ Department of Physics, Graduate School of Science, The University of Tokyo, Tokyo 113-0033, Japan \\ ${ }^{6}$ School of Nuclear Science and Technology, Lanzhou University, Lanzhou 730000, China \\ ${ }^{7}$ State Key Laboratory of Nuclear Physics and Technology, School of Physics, Peking University, Beijing 100871, China \\ ${ }^{8}$ School of Physics and Nuclear Energy Engineering, Beihang University, Beijing 100191, China \\ ${ }^{9}$ Department of Physics, University of Stellenbosch, Stellenbosch 7602, South Africa
}

(Received 23 April 2016; revised manuscript received 14 February 2017; published 3 April 2017)

\begin{abstract}
The self-consistent quasiparticle random-phase approximation (QRPA) approach is formulated in the canonical single-nucleon basis of the relativistic Hatree-Fock-Bogoliubov (RHFB) theory. This approach is applied to study the isobaric analog states (IASs) and Gamow-Teller resonances (GTRs) by taking $\mathrm{Sn}$ isotopes as examples. It is found that self-consistent treatment of the particle-particle residual interaction is essential to concentrate the IAS in a single peak for open-shell nuclei and the Coulomb exchange term is very important to predict the IAS energies. For the GTR, the isovector pairing can increase the calculated GTR energy, while the isoscalar pairing has an important influence on the low-lying tail of the Gamow-Teller transition.
\end{abstract}

DOI: 10.1103/PhysRevC.95.044301

\section{INTRODUCTION}

Exotic nuclei far from the $\beta$-stability line have become an active field of research, as lots of radioactive-ion-beam (RIB) facilities are operating, being upgraded, under construction, or plan to be constructed [1-6]. The charge-exchange excitations of these nuclei play important roles in nuclear physics and various other branches of physics, notably astrophysics. The charge-exchange excitations provide an important probe for studying the spin and isospin properties of the in-medium nuclear interaction. The neutron skin thickness, a basic and critical quantity in nuclear structure, can also be extracted from the sum-rule strengths of the spin-dipole excitations [7]. Moreover, the isobaric analog states (IAS) can be used to study the isospin corrections for the superallowed $\beta$ decays $[8,9]$ and hence to test unitarity of the Cabibbo-KobayashiMaskawa matrix. Furthermore, the properties of chargeexchange excitations are essential to predict many nuclear inputs of astrophysics, such as the nuclear $\beta$-decay halflives, neutrino-nucleus cross sections, and electron-capture cross sections [10-13]. Therefore, nuclear charge-exchange excitations have become one of the hottest topics in nuclear physics and astrophysics.

Charge-exchange excitations can be explored with the charge-exchange reactions, such as $(p, n)$ or $\left({ }^{3} \mathrm{He}, t\right)$ reactions, and the weak-decay processes, such as $\beta$ decays [14-16]. Although the measurement of charge-exchange excitations has achieved great progress in recent years, their theoretical studies

\footnotetext{
*haozhao.liang@riken.jp

${ }^{\dagger}$ mengj@pku.edu.cn
}

are still essential to understand the microscopic mechanism and are also indispensable to many astrophysical applications. Two types of microscopic approaches are widely used in the theoretical investigations of charge-exchange excitations: the shell model and the quasiparticle random-phase approximation (QRPA) approach. Due to the limitation of large configuration space, shell-model calculations are still not feasible for the heavy nuclei away from the magic numbers [10,17-19]. However, the QRPA approach can be applied to all nuclei except a few very light systems.

The QRPA approach can be formulated based on the mean-field basis predicted with the empirical potential, such as the deformed Nilsson model [20-22], the finite-range droplet model with a folded Yukawa single-particle potential [23,24], and the Woods-Saxon potential $[25,26]$. The QRPA approach has also been developed within the finite Fermi system theory [27]. In addition, based on the Skyrme Hatree-Fock (HF) model, RPA calculations were developed for charge-exchange excitations 30 years ago $[28,29]$ and have been extended to the QRPA approach by including pairing correlations to better describe the charge-exchange excitations of open-shell nuclei [30,31]. However, the residual interactions used in these QRPA approaches are not directly derived from the interactions used to obtain the mean-field basis. It has been found that the self-consistency of the QRPA approach is important to describe the Gamow-Teller strength function $[11,12,32,33]$, so the self-consistent QRPA approach has received more and more attention in recent years. Self-consistent QRPA approaches have been developed based on the Skyrme HF + BCS model [34,35] and the Skyrme Hatree-Fock-Bogoliubov (HFB) model [11]. Recently, the self-consistent QRPA approach has been extended to study the charge-exchange excitations and 
$\beta$-decay half-lives of deformed neutron-rich nuclei [36-38], where the finite amplitude method $[39,40]$ was employed for the solution of QRPA in Refs. [37,38]. Moreover, the important ingredient of nuclear force- the tensor force-was found to play a crucial role in describing the nuclear charge-exchange excitations and $\beta$-decay half-lives within the RPA approach $[41,42]$, which inspires much interest to explore the nature of the nuclear tensor force [43]. In addition, the isoscalar protonneutron pairing interaction has also been found to be very important to describe the nuclear charge-exchange excitations and $\beta$-decay half-lives, e.g., in Refs. $[11,44,45]$, which was usually neglected in early studies, e.g., in Refs. [20,24].

During the past years, the covariant density functional theory has successfully described many nuclear phenomena $[4,5,46-49]$ and their predictions are also successfully applied to the simulations of rapid neutron-capture process ( $r$ process) [50-52]. The self-consistent RPA approach was first developed based on the relativistic Hartree (RH) model [53]. The negative-energy states in the Dirac sea are found to be very important to construct the RPA configuration space, which remarkably influences the isoscalar strength distributions [54] and the sum rule of Gamow-Teller (GT) transitions [55]. Furthermore, the QRPA approach is formulated in the canonical single-nucleon basis of the relativistic Hartree-Bogoliubov (RHB) theory and used to study nuclear multipole excitations of open-shell nuclei [56]. The RHB + QRPA approach is then extended to study nuclear charge-exchange excitations $[44,57]$ and further to calculate $\beta$-decay half-lives not only for neutronrich nuclei $[45,58,59]$ but also for the neutron-deficient nuclei [60]. Recently, a systematic calculation on nuclear $\beta$-decay properties, including half-lives, $\beta$-delayed neutron emission probabilities, and the average number of emitted neutrons, was performed with the RHB + QRPA model for 5409 nuclei in the neutron-rich region of the nuclear chart [61].

For the QRPA approaches in the relativistic Hartree approximation, the isovector $\pi$ meson plays an important role in the description of nuclear charge-exchange resonances, while this degree of freedom is absent in the ground-state description due to parity conservation. To account for the contact interaction coming from the pseudovector pion-nucleon coupling, a zerorange counter term is introduced, while its strength is treated as an adjustable parameter to reproduce experimental data on the GT excitation energies. In the relativistic HF (RHF) approximation, the contributions of $\pi$ meson to the nuclear ground-state properties can be naturally included via the exchange (Fock) terms and the description of the nucleon effective mass and the nuclear shell structures is improved $[62,63]$. Based on the RHF model, the fully self-consistent relativistic RPA (RHF + RPA) approach has been developed. The RHF + RPA model achieves an excellent agreement with the data of Gamow-Teller resonances (GTRs) and spin-dipole resonances (SDRs) in doubly magic nuclei, without any readjustment of the parameters of the covariant energy density functional including the zero-range counter term $[64,65]$.

To provide an accurate and reliable description of openshell nuclei, the pairing correlations have to be treated in the proper way. By combining with the BCS method, the RHF + BCS model has been formulated and it is found that the description of nuclear shell evolution along isotopic chain of $Z=50$ and isotonic chain of $N=82$ can be improved with the presence of the degree of freedom associated with the pion pseudovector coupling $[66,67]$. Extending to the neutron or proton drip line, the pairing gap energy becomes comparable to the nucleon separation energy and the continuum effects can be involved substantially by the pairing correlation. It thus requires a unified description of mean-field and pairing correlations; for instance, within the Bogoliubov scheme $[5,68,69]$. Integrated with the Bogoliubov transformation, the relativistic Hartree-Fock-Bogoliubov (RHFB) theory was developed recently [70] and achieved great success in the description of the exotic nuclei far from the $\beta$-stability line [71-73] and superheavy nuclei [74]. Based on RHFB theory, the self-consistent QRPA (RHFB + QRPA) approach was developed and a systematic study of the $\beta$-decay half-lives of neutron-rich even-even nuclei with $20 \leqslant Z \leqslant 50$ has been performed [52].

In this work, we employ the RHFB + QRPA approach to investigate the charge-exchange excitations, including the IAS and GTR. Special attention is paid to the effect of paring interactions. These results are given in Sec. III. In Sec. II, the basic formulas of RHFB theory and QRPA approach are briefly introduced. Finally, a summary and perspectives are presented in Sec. IV.

\section{THEORETICAL FRAMEWORK}

In this section, the basic formulas of the RHFB theory are first briefly introduced, and then the self-consistent QRPA approach based on the RHFB theory is formulated in the canonical basis of the RHFB framework.

\section{A. Effective Lagrangian density}

The basic ansatz of the RHF theory is a Lagrangian density, where nucleons are described as Dirac particles which interact with each other via the exchange of mesons $(\sigma, \omega, \rho$, and $\pi)$ and the photon $(A)$,

$$
\begin{aligned}
\mathcal{L}= & \bar{\psi}\left[i \gamma^{\mu} \partial_{\mu}-M-g_{\sigma} \sigma-\gamma^{\mu} g_{\omega} \omega_{\mu}+g_{\rho} \gamma^{\mu} \vec{\tau} \cdot \vec{\rho}_{\mu}\right. \\
& \left.-\frac{f_{\pi}}{m_{\pi}} \gamma_{5} \gamma^{\mu} \partial_{\mu} \vec{\pi} \cdot \vec{\tau}+e \gamma^{\mu} \frac{1-\tau_{3}}{2} A_{\mu}\right] \psi \\
& +\frac{1}{2} \partial^{\mu} \sigma \partial_{\mu} \sigma-\frac{1}{2} m_{\sigma}^{2} \sigma^{2}-\frac{1}{4} \Omega^{\mu \nu} \Omega_{\mu \nu}+\frac{1}{2} m_{\omega}^{2} \omega^{\mu} \omega_{\mu} \\
& -\frac{1}{4} \vec{R}^{\mu \nu} \cdot \vec{R}_{\mu \nu}+\frac{1}{2} m_{\rho}^{2} \vec{\rho}^{\mu} \cdot \vec{\rho}_{\mu}+\frac{1}{2} \partial^{\mu} \vec{\pi} \cdot \partial_{\mu} \vec{\pi} \\
& -\frac{1}{2} m_{\pi}^{2} \vec{\pi} \cdot \vec{\pi}-\frac{1}{4} F^{\mu \nu} F_{\mu \nu},
\end{aligned}
$$

where $M$ and $m_{i}(i=\sigma, \omega, \rho$, and $\pi)$ are the masses of the nucleon and mesons, $g_{\sigma}, g_{\omega}, g_{\rho}$, and $f_{\pi}$ are meson-nucleon couplings, respectively. $\Omega_{\mu \nu}, \vec{R}^{\mu \nu}$, and $F_{\mu \nu}$ are the field tensors for the vector mesons $\omega, \rho$, and the photon [5].

Following the standard variational procedure of the Lagrangian density, one can obtain the Euler-Lagrange canonical field equations, which just correspond to the Dirac, KleinGordon, and Proca equations for the nucleon, meson, and 
photon fields, respectively. However, these equations are too difficult to be solved exactly, so one has to treat them with some reasonable approximations, such as the Hartree or Hartree-Fock approximations.

\section{B. Energy functional and Dirac Hartree-Fock equation}

Before applying the Hartree or Hartree-Fock approximations, the energy functional should be first built up, which is obtained by taking the expectation value of the Hamiltonian. The Hamiltonian $H$ in the nucleon space can be expressed as

$$
\begin{aligned}
H= & \int d^{3} x_{1} \bar{\psi}(-i \boldsymbol{\gamma} \cdot \nabla+M) \psi+\frac{1}{2} \iint d^{3} x_{1} d^{4} x_{2} \\
& \times \sum_{\substack{i=\sigma, \omega, \rho, \pi, A}} \bar{\psi}\left(x_{1}\right) \bar{\psi}\left(x_{2}\right) \Gamma_{i}(1,2) D_{i}(1,2) \psi\left(x_{2}\right) \psi\left(x_{1}\right),
\end{aligned}
$$

where the two-body interaction vertices $\Gamma_{i}(1,2)$ for the meson and photon fields are

$$
\begin{aligned}
& \Gamma_{\sigma}(1,2)=-g_{\sigma}(1) g_{\sigma}(2), \\
& \Gamma_{\omega}(1,2)=+g_{\omega}(1) \gamma_{\mu}(1) g_{\omega}(2) \gamma^{\mu}(2), \\
& \Gamma_{\rho}(1,2)=+g_{\rho}(1) \gamma_{\mu}(1) \vec{\tau}(1) \cdot g_{\rho}(2) \gamma^{\mu}(2) \vec{\tau}(2), \\
& \Gamma_{\pi}(1,2)=-\left[\frac{f_{\pi}}{m_{\pi}} \vec{\tau} \gamma_{5} \gamma_{\mu} \partial^{\mu}\right]_{1} \cdot\left[\frac{f_{\pi}}{m_{\pi}} \vec{\tau} \gamma_{5} \gamma_{\nu} \partial^{\nu}\right]_{2}, \\
& \Gamma_{A}(1,2)=+\frac{e^{2}}{4}\left[\gamma_{\mu}\left(1-\tau_{3}\right)\right]_{1}\left[\gamma^{\mu}\left(1-\tau_{3}\right)\right]_{2} .
\end{aligned}
$$

$D_{i}(1,2)$ are the propagators of the meson and photon fields, which are usually simplified by neglecting retardation effects.

To quantize the Hamiltonian $H$ in Eq. (2), the nucleon field operators $\psi$ and $\bar{\psi}$ are expanded on the set of creation and annihilation operators of nucleons in the no-sea approximation. Furthermore, the trial ground state $\left|\Phi_{0}\right\rangle$ is chosen as a Slater determinant in the Hartree-Fock approximation. The energy functional is then obtained from the expectation with respect to the ground state $\left|\Phi_{0}\right\rangle$,

$$
E=\left\langle\Phi_{0}|H| \Phi_{0}\right\rangle=\left\langle\Phi_{0}\left|\left(T+\sum_{i} V^{i}\right)\right| \Phi_{0}\right\rangle,
$$

where $T$ and $V^{i}$ are the kinetic term and two-body interaction term, respectively. The expectation of the two-body interaction term $V^{i}$ will lead to two types of contributions; namely, the direct (Hartree) and exchange (Fock) terms. With only the direct term, Eq. (8) just corresponds to the energy functional of the RMF or RH theory, while with both direct and exchange terms, one obtains the energy functional of the RHF theory.

Taking the variation of the energy functional (8) with respect to the Dirac spinor $f_{\alpha}$, one then gets the Dirac Hartree-Fock equation,

$$
\int d \boldsymbol{r}^{\prime} h\left(\boldsymbol{r}, \boldsymbol{r}^{\prime}\right) f_{\alpha}\left(\boldsymbol{r}^{\prime}\right)=\varepsilon_{\alpha} f_{\alpha}(\boldsymbol{r}),
$$

where $h\left(\boldsymbol{r}, \boldsymbol{r}^{\prime}\right)$ is the single-particle Dirac Hamiltonian and $\varepsilon$ is the single-particle energy including the rest mass. There are three parts for $h\left(\boldsymbol{r}, \boldsymbol{r}^{\prime}\right)$; i.e., $h=h^{\mathrm{kin}}+h^{\mathrm{D}}+h^{\mathrm{E}}$. They respectively denote the kinetic energy, the direct local potential, and the exchange nonlocal potential. The readers can refer to Ref. [70] for the detailed expressions of $h^{\mathrm{kin}}, h^{\mathrm{D}}$, and $h^{\mathrm{E}}$.

\section{Relativistic Hartree-Fock-Bogoliubov theory}

To describe the properties of open-shell nuclei, the pairing correlations should be included, which is taken into account with the Bogoliubov theory in this work. Following the standard procedure of the Bogoliubov transformation [75-77], one then obtain the relativistic Hartree-Fock-Bogoliubov equation as

$$
\begin{gathered}
\int d \boldsymbol{r}^{\prime}\left(\begin{array}{cc}
h\left(\boldsymbol{r}, \boldsymbol{r}^{\prime}\right) & \Delta\left(\boldsymbol{r}, \boldsymbol{r}^{\prime}\right) \\
\Delta\left(\boldsymbol{r}, \boldsymbol{r}^{\prime}\right) & -h\left(\boldsymbol{r}, \boldsymbol{r}^{\prime}\right)
\end{array}\right)\left(\begin{array}{l}
f_{U}\left(\boldsymbol{r}^{\prime}\right) \\
f_{V}\left(\boldsymbol{r}^{\prime}\right)
\end{array}\right) \\
=\left(\begin{array}{cc}
E+\lambda & 0 \\
0 & E-\lambda
\end{array}\right)\left(\begin{array}{l}
f_{U}(\boldsymbol{r}) \\
f_{V}(\boldsymbol{r})
\end{array}\right),
\end{gathered}
$$

where $f_{U}$ and $f_{V}$ are the quasiparticle spinors and $\lambda$ is the chemical potential. The pairing potential $\Delta\left(\boldsymbol{r}, \boldsymbol{r}^{\prime}\right)$ can be expressed as

$$
\Delta\left(\boldsymbol{r}, \boldsymbol{r}^{\prime}\right)=-\frac{1}{2} \sum_{\beta} V_{\alpha \beta}^{p p}\left(\boldsymbol{r}, \boldsymbol{r}^{\prime}\right) \kappa_{\beta}\left(\boldsymbol{r}, \boldsymbol{r}^{\prime}\right),
$$

where $\kappa_{\beta}\left(\boldsymbol{r}, \boldsymbol{r}^{\prime}\right)$ is the pairing tensor. For the pairing interaction $V^{p p}$, we adopt the pairing part of the Gogny force,

$$
\begin{aligned}
V^{p p}\left(\boldsymbol{r}, \boldsymbol{r}^{\prime}\right)= & \sum_{i=1,2} e^{\left[\left(\boldsymbol{r}-\boldsymbol{r}^{\prime}\right) / \mu_{i}\right]^{2}}\left(W_{i}+B_{i} P^{\sigma}\right. \\
& \left.-H_{i} P^{\tau}-M_{i} P^{\sigma} P^{\tau}\right),
\end{aligned}
$$

with the set D1S [78] for the parameters $\mu_{i}, W_{i}, B_{i}, H_{i}$, and $M_{i}$.

In this work, the spherical symmetry is assumed for the nuclear systems and the RHFB equation is solved by an expansion of quasiparticle spinors in the Dirac Woods-Saxon (DWS) basis [70,79]. The numbers of positive- and negativeenergy states in the DWS basis are taken as $N_{F}=28$ and $N_{D}=20$, respectively. Details of solving the RHFB equations in the DWS basis can be found in Ref. [70].

\section{Quasiparticle random phase approximation}

The QRPA equations can be derived from the timedependent RHFB theory in the limit of small-amplitude oscillations similar to Refs. [44,56]. Previous studies have found that the QRPA equations can be easily solved in the canonical basis, in which the RHFB wave functions are expressed in the form of BCS-like wave functions. With spherical symmetry, the quasiparticle pairs can be coupled to a good angular momentum and the matrix equations of the QRPA for the charge-exchange excitations read

$$
\left(\begin{array}{cc}
A_{p n p^{\prime} n^{\prime}}^{J} & B_{p n p^{\prime} n^{\prime}}^{J} \\
-B_{p n p^{\prime} n^{\prime}}^{* J} & -A_{p n p^{\prime} n^{\prime}}^{* J}
\end{array}\right)\left(\begin{array}{c}
X_{p^{\prime} n^{\prime}}^{\lambda J} \\
Y_{p^{\prime} n^{\prime}}^{\lambda J}
\end{array}\right)=E_{\lambda}\left(\begin{array}{c}
X_{p n}^{\lambda J} \\
Y_{p n}^{\lambda J}
\end{array}\right),
$$

where $p, p^{\prime}$, and $n, n^{\prime}$ denote proton and neutron quasiparticle canonical states, respectively. For each transition energy $E_{\lambda}$, the quantities $X_{p n}^{\lambda J}$ and $Y_{p n}^{\lambda J}$ denote the corresponding forward- and backward-going QRPA amplitudes, respectively. 
The angular-momentum coupled matrix elements $A^{J}$ and $B^{J}$ read

$$
\begin{aligned}
A_{p n p^{\prime} n^{\prime}}^{J}= & H_{p p^{\prime}}^{11} \delta_{n n^{\prime}}+H_{n n^{\prime}}^{11} \delta_{p p^{\prime}} \\
& +H_{p n p^{\prime} n^{\prime}}^{p h J}\left(u_{p} v_{n} u_{p^{\prime}} v_{n^{\prime}}+v_{p} u_{n} v_{p^{\prime}} u_{n^{\prime}}\right) \\
& +H_{p n p^{\prime} n^{\prime}}^{p p J}\left(u_{p} u_{n} u_{p^{\prime}} u_{n^{\prime}}+v_{p} v_{n} v_{p^{\prime}} v_{n^{\prime}}\right) \\
B_{p n p^{\prime} n^{\prime}}^{J}= & H_{p n p^{\prime} n^{\prime}}^{p h J}\left(u_{p} v_{n} v_{p^{\prime}} u_{n^{\prime}}+v_{p} u_{n} u_{p^{\prime}} v_{n^{\prime}}\right) \\
& -H_{p n p^{\prime} n^{\prime}}^{p p J}\left(u_{p} u_{n} v_{p^{\prime}} v_{n^{\prime}}+v_{p} v_{n} u_{p^{\prime}} u_{n^{\prime}}\right)
\end{aligned}
$$

with

$$
H_{k k^{\prime}}^{11}=h_{k k^{\prime}}\left(u_{k} u_{k^{\prime}}-v_{k} v_{k^{\prime}}\right)-\Delta_{k k^{\prime}}\left(u_{k} v_{k^{\prime}}+v_{k} u_{k^{\prime}}\right) .
$$

The terms $H^{p h J}$ and $H^{p p J}$ in matrix elements $A^{J}$ and $B^{J}$ denote the contributions from particle-hole (ph) and particleparticle (pp) interactions, respectively.

In the self-consistent QRPA approach based on the RHFB theory, the contributions from exchange terms must be included, so the term $H^{p h J}$ corresponding to the ph interaction $V^{p h}$ is

$$
H_{p n p^{\prime} n^{\prime}}^{p h J}=V_{p n^{\prime} n p^{\prime}}^{p h J}-V_{p n^{\prime} p^{\prime} n^{p}}^{p h J} .
$$

In this work, $V^{p h}$ includes the contributions from the $\sigma-, \omega$-, $\rho$-, and $\pi$-meson fields, i.e.,

$$
V^{p h}=\sum_{i=\sigma, \omega, \rho, \pi} \Gamma_{i}(1,2) D_{i}(1,2),
$$

where $\Gamma_{i}(1,2)$ and $D_{i}(1,2)$ are the interaction vertices and propagators of corresponding meson fields given in Sec. II B. In addition, a zero-range pionic counter term should be included to cancel the contact interaction coming from the pion pseudovector coupling, which reads

$$
V_{\pi}^{\delta}(1,2)=-\frac{1}{3}\left[\frac{f_{\pi}}{m_{\pi}} \vec{\tau} \gamma_{5} \gamma_{i}\right]_{1} \cdot\left[\frac{f_{\pi}}{m_{\pi}} \vec{\tau} \gamma_{5} \gamma^{i}\right]_{2} \delta\left(\boldsymbol{r}_{1}-\boldsymbol{r}_{2}\right) .
$$

Similarly, the term $H^{p p J}$ corresponding to the pp interaction $V^{p p}$ is

$$
H_{p n p^{\prime} n^{\prime}}^{p p J}=V_{p n p^{\prime} n^{\prime}}^{p p J}-V_{p n n^{\prime} p^{\prime}}^{p p J} .
$$

In the isovector $(T=1) \mathrm{pp}$ channel, we adopt the pairing part of the Gogny force with the parameter set D1S as in the RHFB ground-state calculations. In the isoscalar ( $T=$ $0) \mathrm{pp}$ channel, we employ a finite-range interaction as in Refs. [11,44,45,52,58-60],

$$
V_{T=0}^{p p}(1,2)=-V_{0} \sum_{i=1,2} g_{i} e^{\left[\left(\boldsymbol{r}_{1}-\boldsymbol{r}_{2}\right) / \mu_{i}\right]^{2}} \hat{\Pi}_{S=1, T=0},
$$

with $\mu_{1}=1.2 \mathrm{fm}, \mu_{2}=0.7 \mathrm{fm}, g_{1}=1$, and $g_{2}=-2$. The operator $\hat{\Pi}_{S=1, T=0}$ projects onto states with $S=1$ and $T=0$. For the strength parameter $V_{0}$, we employ the following ansatz proposed in Ref. [52]:

$$
V_{0}=V_{L}+\frac{V_{D}}{1+e^{a+b(N-Z)}},
$$

with $V_{L}=134.0 \mathrm{MeV}, V_{D}=121.1 \mathrm{MeV}, a=8.5$, and $b=$ -0.4 adjusted to obtain the best possible description of available half-life data [80] in the region $20 \leqslant Z \leqslant 50$.

By diagonalizing the QRPA matrix in Eq. (13), one can get the discrete transition energies $E_{\lambda}$ and the corresponding QRPA amplitudes $X_{p n}^{\lambda J}$ and $Y_{p n}^{\lambda J}$. Then the transition probabilities $B_{\lambda J}$ induced by the operator $T^{J M}$ between the ground state of the even-even $(N, Z)$ nucleus and the excited state of the odd-odd $(N+1, Z-1)$ or $(N-1, Z+1)$ nucleus can be calculated by

$$
B_{\lambda}=\left|\sum_{p n}\left\langle p\left\|T^{J}\right\| n\right\rangle\left[X_{p n}^{\lambda J} u_{p} v_{n}+(-1)^{J} Y_{p n}^{\lambda J} v_{p} u_{n}\right]\right|^{2} .
$$

The strength distribution is obtained by folding the discrete transition probabilities with Lorentzian function, i.e.,

$$
R(E)=\sum_{\lambda} B_{\lambda} \frac{\Gamma / 2 \pi}{\left(E-E_{\lambda}\right)^{2}+\Gamma^{2} / 4},
$$

where the width $\Gamma$ is taken to be $1 \mathrm{MeV}$ for illustrating our calculations of the spin-isospin excitations.

\section{RESULTS AND DISCUSSION}

In the self-consistent QRPA calculations, a reasonable description of nuclear ground-state properties is essential to predict nuclear charge-exchange excitations. Therefore, in this section, we first study the description of nuclear ground-state properties by using the RHFB theory. Nuclear masses, $Q_{\beta}$ values, two-neutron separation energies, and the neutron-skin thicknesses are taken as examples. The self-consistent QRPA calculations based on RHFB theory are then shown for the IAS and GTR, on which the effects of the ph and pp residual interactions will be investigated carefully. The effective interactions PKO1 [62] and DD-ME2 [81] are adopted for the RHFB (+QRPA) and RHB (+QRPA) calculations, respectively.

\section{A. Ground-state properties}

Nuclear mass is a very important property of nucleus, and it can be used to determine various reaction energies and decay energies. Figure 1 gives the deviations of the theoretical nuclear masses from the experimental data [82] for the eveneven $\mathrm{Ca}, \mathrm{Ni}$, and $\mathrm{Sn}$ isotopes. In general, the calculations with PKO1 and DD-ME2 reproduce the experimental data within $2 \mathrm{MeV}$ and the results with PKO1 are slightly better than those with DD-ME2. The PKO1 generally overestimates the nuclear masses except for the Ni isotopes, while the DD-ME2 generally underestimates the nuclear masses. Qualitatively, the root-mean-square (rms) deviations and mean deviations between the mass predictions and experimental data for nuclei shown in Fig. 1 are 1.51 and $0.59 \mathrm{MeV}$ for the PKO1, and are 2.01 and $-1.15 \mathrm{MeV}$ for DD-ME2, respectively.

Figure 2 shows $Q_{\beta}$ values of the even-even $\mathrm{Ca}, \mathrm{Ni}$, and $\mathrm{Sn}$ isotopes. For stable nuclei, we present the isobaric mass differences calculated with the same formula as that of $Q_{\beta}$ calculations for $\beta$-unstable nuclei. It is clear that $Q_{\beta}$ values calculated with PKO1 are similar to those calculated with 


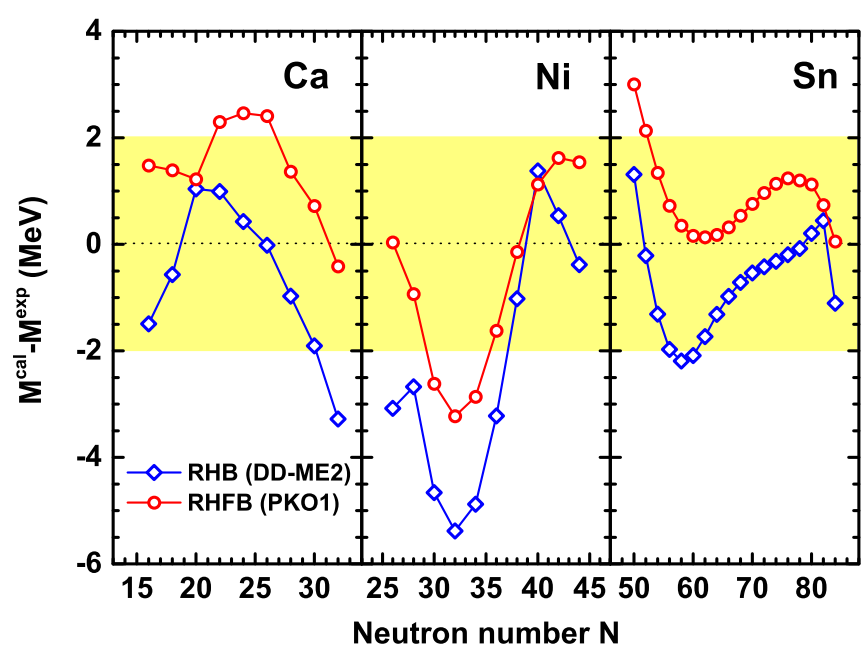

FIG. 1. Deviations of the theoretical nuclear masses from the experimental data [82] for the even-even $\mathrm{Ca}, \mathrm{Ni}$, and $\mathrm{Sn}$ isotopes. The theoretical results are calculated by the RHFB theory with the effective interaction PKO1 (open circles) or the RHB theory with the effective interaction DD-ME2 (open diamonds).

DD-ME2, although there are certain differences between mass predictions with PKO1 and DD-ME2. Comparing with the experimental data, both PKO1 and DD-ME2 generally underestimate experimental $Q_{\beta}$ values. This indicates that the half-lives of these magic nuclei would be overestimated if there are no further improvements, such as the inclusion of isoscalar $\mathrm{p} n$ residual pairing [52].

Neutron separation energy is another important nuclear property, which contains detailed information about the nuclear structure. Figure 3 shows the two-neutron separation energies $S_{2 n}$ of the even-even $\mathrm{Ca}, \mathrm{Ni}$, and $\mathrm{Sn}$ isotopes calculated by the RHFB theory. It is clear that the RHFB approach well reproduces the experimental data in a rather

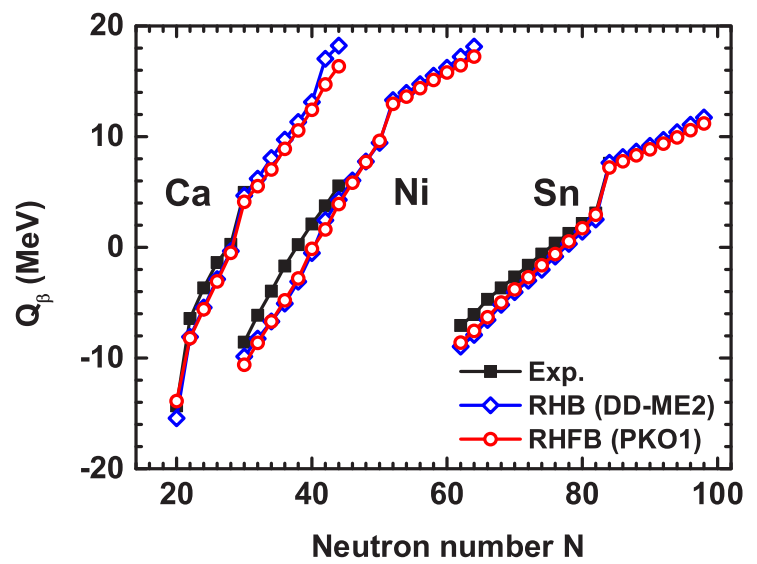

FIG. 2. $Q_{\beta}$ values of the even-even $\mathrm{Ca}, \mathrm{Ni}$, and $\mathrm{Sn}$ isotopes (isobaric mass differences for stable nuclei). The RHFB calculations with PKO1 are denoted by the open circles. For comparison, the experimental data [82] and the calculated results by the RHB theory with DD-ME2 are shown by the filled squares and open diamonds, respectively.

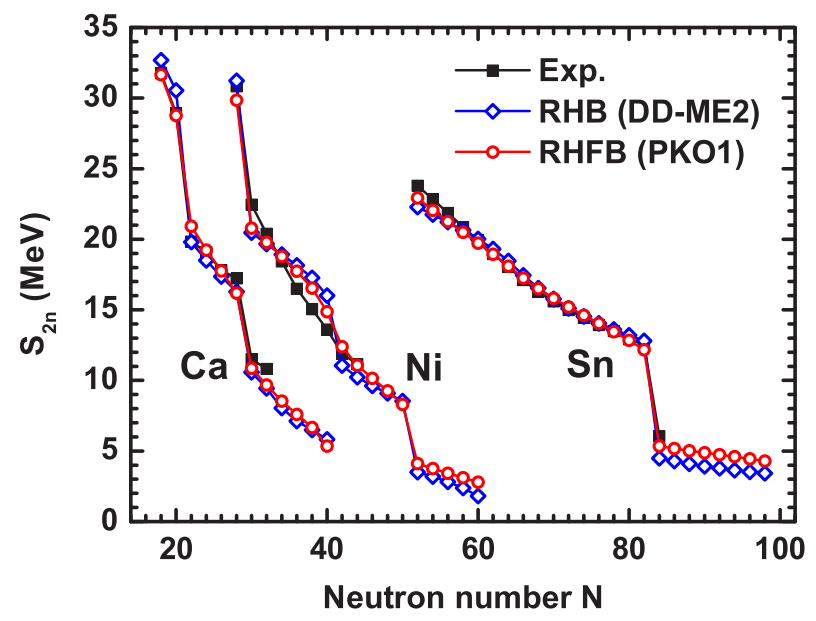

FIG. 3. Two-neutron separation energies of the even-even $\mathrm{Ca}$, $\mathrm{Ni}$, and $\mathrm{Sn}$ isotopes. The RHFB calculations with the effective interaction PKO1 are denoted by the open circles. For comparison, the experimental data [82] and the results calculated by RHB theory with the effective interaction DD-ME2 are shown by the filled squares and open diamonds, respectively.

wide range from $Z=20$ to $Z=50$. It is known that the abrupt drop of $S_{2 n}$ generally reflects the existence of shell structure. From the abrupt drop of experimental $S_{2 n}$ in Fig. 3, the shell structures at $N=20,28$, and 82 are clearly observed. Both the RHB and RHFB approaches correctly describe the positions of the shell structures. However, the RHB calculations with the effective interaction DD-ME2 overestimate the shell effects at $N=40$ for the Ni isotopes. For the RHFB calculations with the effective interaction PKO1, the strengthes of the shell closures at $N=20,28$, and 82 are generally well reproduced, as well as the shell effects at $N=40$.

The neutron-skin thicknesses of the even-even $\mathrm{Sn}$ isotopes are shown in Fig. 4. The calculations with PKO1 reproduce the experimental results from SDR except for ${ }^{114} \mathrm{Sn}$, and the calculations with DD-ME2 generally reproduce the experimental results from the three methods presented in Fig. 4. Comparing between these two approaches, the results of PKO1 are systematically larger than those of DD-ME2. This can be mainly explained by the larger symmetry energy of PKO1 $E_{\text {sym }}=$ $34.4 \mathrm{MeV}$ comparing with that of DD-ME2 $E_{\mathrm{sym}}=32.3 \mathrm{MeV}$, since there exists a linear relation between the neutron-skin thickness and the symmetry energy of nuclear matter at saturation density [85]. Significant progress has been made on constraining the symmetry energy during the past decades. A recent study summarized the current available constraints on the symmetry energy from various methods are in agreement with $E_{\text {sym }}=32.5 \pm 2.5 \mathrm{MeV}$ [86]. For that, the symmetry energies of both PKO1 and DD-ME2 still agree with these constraints.

\section{B. Spin-isospin excitations}

As a first test of the present QRPA model, we perform the so-call IAS check to verify the model self-consistency. If the Coulomb interaction is switched off, the nuclear Hamiltonian 


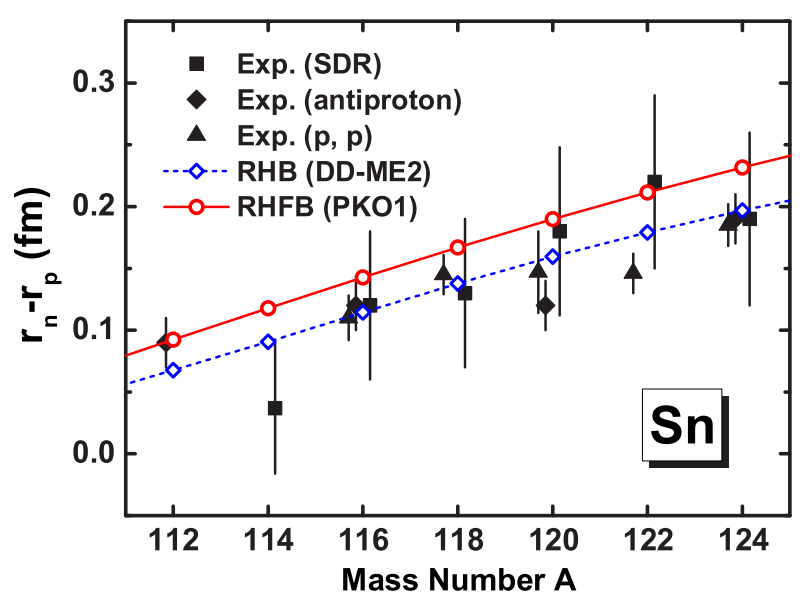

FIG. 4. Neutron-skin thicknesses $\left(r_{n}-r_{p}\right)$ of the even-even Sn isotopes. Open circles and open diamonds show the results calculated by the RHFB theory with PKO1 and the RHB theory with DD-ME2, respectively. The experimental results from the spin-dipole resonance (SDR) [7], antiprotonic x-ray data [83], and proton elastic scattering [84] are shown by the filled squares, diamonds, and triangles, respectively.

would commute with the isospin-lowering $T_{-}$and -raising $T_{+}$operators and then the IAS should be degenerate with its isobaric multiplet partners. This degeneracy is broken by the mean-field approximation, while it can be restored by the self-consistent RPA calculation [87]. Taking the IAS in ${ }^{114} \mathrm{Sn}$ as an example, the corresponding transition probabilities are shown in Fig. 5, which are calculated by the RHFB + QRPA approach without the Coulomb interaction. It is found that the unperturbed excitations mainly locate between $E=-5$ and $-4 \mathrm{MeV}$, which indicates the isospin symmetry breaking in the RHFB theory. By including the ph residual interactions in the

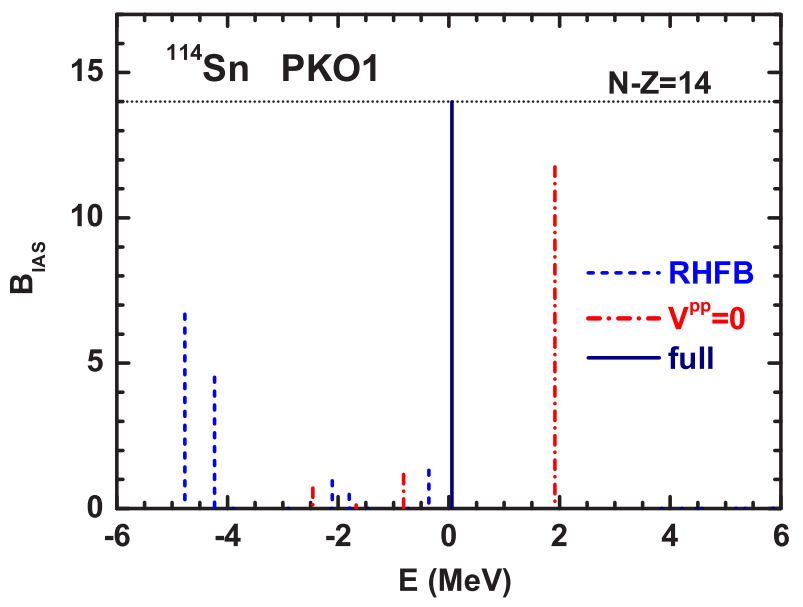

FIG. 5. Transition probabilities for the IAS in ${ }^{114} \mathrm{Sn}$. The calculations are performed by the RHFB + QRPA approach with PKO1, while the Coulomb interaction is switched off. The horizontal dotted line denotes the $N-Z$ sum rule. For comparison, the unperturbed result (labeled by RHFB) and the calculation without the pp residual interaction $\left(V^{p p}=0\right)$ are shown by the dashed and dash-dotted lines, respectively.

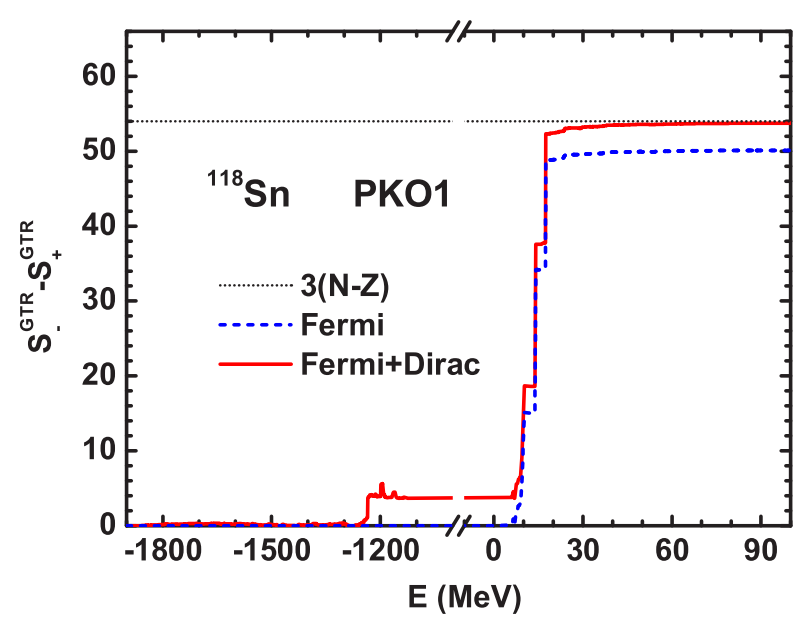

FIG. 6. Running sum of the GT transition probabilities for ${ }^{118} \mathrm{Sn}$ calculated by the RHFB + QRPA approach with PKO1. The dashed line shows the QRPA calculation with only the ph configurations from the Fermi states. The solid line corresponds to the calculation further including the configurations from the occupied Fermi states and the unoccupied Dirac states. The horizontal dotted line corresponds to the value $3(N-Z)$ of the Ikeda sum rule.

QRPA approach, the transition energy with the largest strength increases to $E=1.9 \mathrm{MeV}$, while it still remarkably departs from zero. Furthermore, when the pp residual interactions are included, the energy of IAS goes to $0.05 \mathrm{MeV}$ and it also exhausts $99.94 \%$ of the $N-Z$ sum rule. This indicates the self-consistency is well preserved in the present RHFB + QRPA approach only when the $\mathrm{ph}$ and pp residual interactions are both taken into account in the QRPA calculations.

As a step further, the sum rule of GT transition probabilities is employed to check the QRPA model. Figure 6 presents the running sum of the GT transition probabilities by taking ${ }^{118} \mathrm{Sn}$ as an example, which is defined to be

$$
\left(S_{-}^{\mathrm{GTR}}-S_{+}^{\mathrm{GTR}}\right)_{E}=\sum_{E_{\lambda}<E}\left(B_{\lambda}^{-}-B_{\lambda}^{+}\right),
$$

where $E_{\lambda}$ represent the GT transition energies and $B_{\lambda}^{ \pm}$are the corresponding transition probabilities in the $T_{ \pm}$channels. When the complete set of states is included, Eq. (25) gives the value $3(N-Z)$ of the Ikeda sum rule [88]. In the relativistic framework, it has been found that the total GT strength in the nucleon sector is reduced by about $12 \%$ in nuclear matter [89] and by $6 \% \sim 7 \%$ in finite nuclei $[55,64]$ when compared to the Ikeda sum rule, if the effects related to the Dirac sea are neglected. The dashed line in Fig. 6 presents the running sum of the GT transition probabilities calculated with only the ph configurations from the Fermi states. The value of $\left(S_{-}^{\mathrm{GTR}}-\right.$ $S_{+}^{\text {GTR }}$ ) only goes to about 50 even the sum is extended up to $E=100 \mathrm{MeV}$, which is about 7\% less than the Ikeda sum rule. When the ph configurations from the occupied Fermi states and the unoccupied Dirac states are further included, they contribute about four to the sum rule even the sum only goes to $E=-1000 \mathrm{MeV}$, and this value just compensates the above missing part. This confirms that the total sum rule $3(N-Z)$ is exhausted only when the configurations from the occupied 


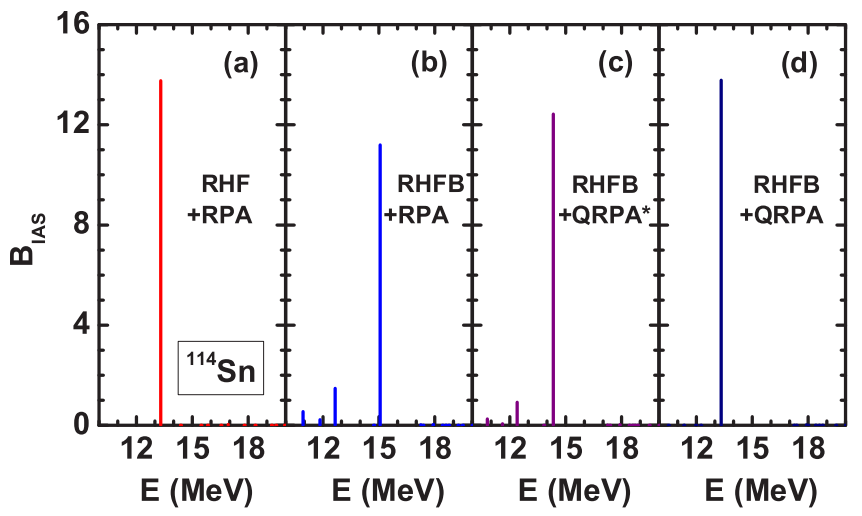

FIG. 7. Transition probabilities for the IAS in ${ }^{114} \mathrm{Sn}$ calculated with PKO1. The RHF + RPA, RHFB+RPA, RHFB + QRPA*, and RHFB + QRPA calculations are shown in panels (a)-(d), respectively. See the text for details.

Fermi states and the unoccupied Dirac states are included. Therefore, all the following calculations strictly include these configurations.

The IAS is the simplest but important charge-exchange excitation mode and it has been observed in experiments with a single peak with a narrow width [90]. It has been found that the consistent treatment of pairing correlations in QRPA calculations plays an essential role in concentrating the IAS in a single peak $[34,44]$. To investigate such a fact in the RHFB + QRPA approach, Fig. 7 gives the calculated transition probabilities for the IAS in ${ }^{114} \mathrm{Sn}$.

In Fig. 7(a), the results calculated without any pairing interaction are shown and a single peak is observed. In a sense, the treatment of pairing is consistent here because it is not included in both the ground-state and IAS calculations, but the pairing correlations are essential for open-shell nuclei. The pairing is then included in the RHFB calculation for the ground-state properties, while the pp residual interaction is excluded in the QRPA calculation, which is shown in Fig. 7(b). It is found that the calculated transition probabilities become fragmented, inconsistent with the experimentally observed single narrow resonance. In addition, the main peak is shifted to higher excitation energy. Furthermore, the direct part of the pp residual interaction is included in the QRPA calculation, and the corresponding results are shown in Fig. 7(c). The fragmentation of IAS still exists although it has been partially eliminated. In Fig. 7(d), the fully self-consistent RHFB + QRPA calculation is presented. The IAS is again collected in a single peak, which can exhaust $98 \%$ of the $N-Z$ sum rule. Therefore, the consistent treatment of pairing correlations in the QRPA calculation is essential to concentrate the IAS in a single peak, and hence the pp residual interaction has to be incorporated for better understanding the IAS transitions of open-shell nuclei.

The IAS excitation energies of the even-even $\mathrm{Sn}$ isotopes are shown in Fig. 8. To investigate the influence of pairing interaction and exchange terms of mean fields, the calculations with the self-consistent RHF + RPA and RHB + QRPA approaches are also shown in addition to the



FIG. 8. IAS excitation energies of the even-even $\mathrm{Sn}$ isotopes. The experimental data [90] are denoted by the filled squares. The self-consistent RHF + RPA and RHFB + QRPA calculations with $\mathrm{PKO} 1$ are shown by the open and filled circles, respectively, while the self-consistent RHB + QRPA calculations with DD-ME2 are shown by the filled diamonds. For comparison, the results obtained with RHFB + QRPA approach with PKO1 but excluding the Coulomb exchange term are denoted by the open squares.

results from the self-consistent RHFB + QRPA calculations. Comparing the results of the self-consistent RHF + RPA and RHFB + QRPA calculations, it is found that the inclusion of $T=1$ pairing interactions can slightly increase the calculated IAS excitation energies. Moreover, it is found that the IAS excitation energies calculated with the RHFB + QRPA and RHB + QRPA approaches are about 300 and $600 \mathrm{keV}$ lower than the experimental data.

Since the nonzero IAS excitation energy originates from the existence of the Coulomb field, the different treatments of the Coulomb field would play an important role in understanding this systematic discrepancy between RHFB + QRPA and RHB + QRPA. To verify this argument, we further perform the self-consistent RHFB + QRPA calculations while the Coulomb exchange term is switched off from the beginning. The corresponding results are shown by the open squares in Fig. 8. It is seen that these results are almost the same as those of the RHB + QRPA calculations, so the Coulomb exchange term is responsible for the difference between the IAS excitation energies with the RHFB + QRPA and RHB + QRPA approaches, and the proper treatment of the Coulomb field is important to predict the IAS excitation energies.

The GTR is another important mode of charge-exchange excitation and it plays an important role in understanding many nuclear processes in nucleosynthesis, such as nuclear $\beta$ decay and electron-capture process. It has been found that the GTR in the doubly magic nuclei ${ }^{48} \mathrm{Ca},{ }^{90} \mathrm{Zr}$, and ${ }^{208} \mathrm{~Pb}$ are well reproduced based on the RHF + RPA approach without any readjustment of the ph residual interaction [64]. In this work, we will check whether such self-consistence is kept even for the open-shell nuclei. In Fig. 9, the GT strength distribution in ${ }^{118} \mathrm{Sn}$ calculated by the self-consistent RHFB + QRPA 


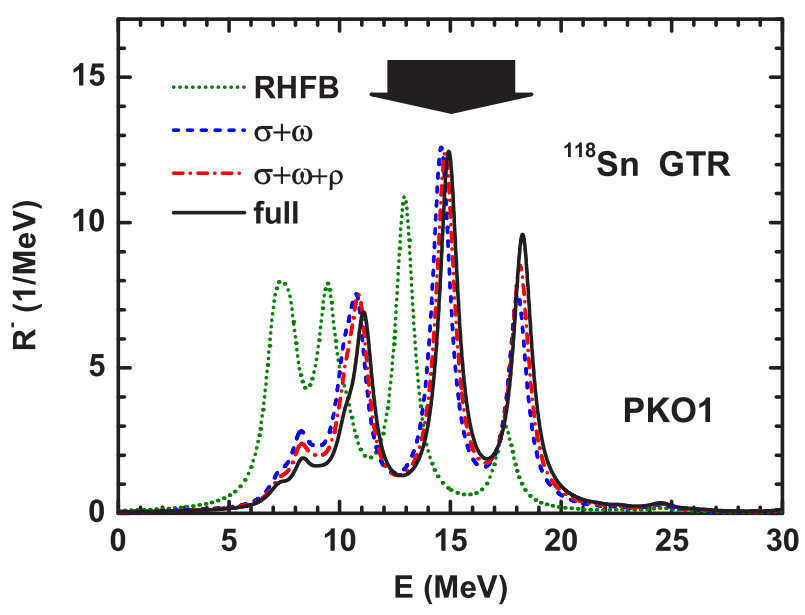

FIG. 9. GT strength distribution in ${ }^{118} \mathrm{Sn}$ calculated by the RHFB + QRPA approach with PKO1. The unperturbed (labeled by RHFB) strength, the calculation with only ph residual interactions of $\sigma$ and $\omega$ fields, and that with only ph residual interactions of $\sigma, \omega$, and $\rho$ fields (excluding $\pi$ field) are shown by the dotted, dashed, and dash-dotted lines, respectively. The experimental data [90] are shown with an arrow, whose width illustrates the width of the resonance.

approach is shown. It is compared with the unperturbed case, the calculation with only ph residual interactions of $\sigma$ and $\omega$ fields, and that with only ph residual interactions of $\sigma$, $\omega$, and $\rho$ fields. It is clear that the $\sigma$ and $\omega$ mesons play the essential role via the exchange terms, while the $\rho$ and $\pi$ mesons only play a minor role. Similar to the case in the doubly magic nuclei, the experimental excitation energy of the main peak of GTR in open-shell nuclei is also well reproduced by the RHFB + QRPA approach without any readjustment of ph residual interactions.

Comparing with the doubly magic nuclei, pairing interaction is essential to describe the properties of open-shell nuclei. Figure 10 presents the effect of the isovector $T=1$ pairing interaction on the GT strength distribution in ${ }^{118} \mathrm{Sn}$. It is seen

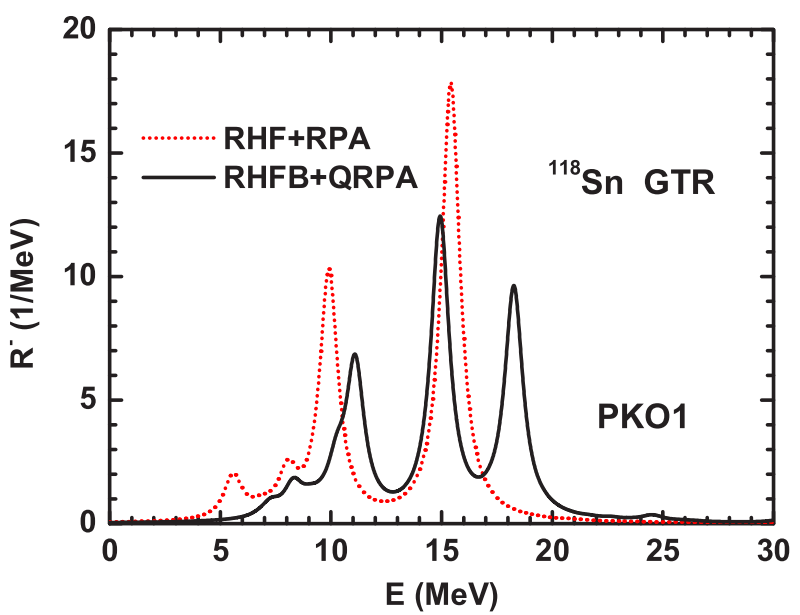

FIG. 10. GT strength distribution in ${ }^{118} \mathrm{Sn}$ calculated by the RHF + RPA (dotted line) and RHFB + QRPA (solid line) approaches with PKO1.
TABLE I. Main neutron-to-proton (Q)RPA amplitudes $\left(X_{p h}^{2}-\right.$ $Y_{p h}^{2}>1 \%$ ) for different GT excitations in ${ }^{118} \mathrm{Sn}$ calculated by the $\mathrm{RHF}+\mathrm{RPA}$ and RHFB + QRPA approaches. Excitation energies are in unit of MeV.

\begin{tabular}{|c|c|c|c|c|c|}
\hline \multirow[t]{2}{*}{ Configurations } & \multicolumn{2}{|c|}{$\mathrm{RHF}+\mathrm{RPA}$} & \multicolumn{3}{|c|}{ RHFB + QRPA } \\
\hline & $E=9.9$ & 15.4 & 11.1 & 14.9 & 18.3 \\
\hline$\nu 1 g_{9 / 2} \rightarrow \pi 1 g_{7 / 2}$ & $5.0 \%$ & $90.5 \%$ & $6.4 \%$ & $82.5 \%$ & $8.4 \%$ \\
\hline$v 1 g_{7 / 2} \rightarrow \pi 1 g_{7 / 2}$ & $10.4 \%$ & $1.3 \%$ & $4.8 \%$ & $1.1 \%$ & \\
\hline$v 1 g_{7 / 2} \rightarrow \pi 2 d_{5 / 2}$ & $2.5 \%$ & & $2.8 \%$ & & \\
\hline$v 2 d_{5 / 2} \rightarrow \pi 2 d_{5 / 2}$ & $12.5 \%$ & & $6.5 \%$ & & \\
\hline$\nu 2 d_{5 / 2} \rightarrow \pi 2 d_{3 / 2}$ & $57.7 \%$ & $2.3 \%$ & $16.7 \%$ & $2.1 \%$ & \\
\hline$\nu 2 d_{3 / 2} \rightarrow \pi 2 d_{5 / 2}$ & $3.3 \%$ & & $1.3 \%$ & & \\
\hline$\nu 2 d_{3 / 2} \rightarrow \pi 2 d_{3 / 2}$ & $6.0 \%$ & & $3.9 \%$ & & \\
\hline$v 2 d_{3 / 2} \rightarrow \pi 3 s_{1 / 2}$ & $1.7 \%$ & & $1.2 \%$ & & \\
\hline$\nu 2 d_{3 / 2} \rightarrow \pi 3 d_{5 / 2}$ & & $1.5 \%$ & & & \\
\hline$v 2 d_{3 / 2} \rightarrow \pi 3 d_{3 / 2}$ & & $1.7 \%$ & & & \\
\hline$v 3 s_{1 / 2} \rightarrow \pi 3 s_{1 / 2}$ & & & $5.7 \%$ & & \\
\hline$v 1 h_{11 / 2} \rightarrow \pi 1 h_{11 / 2}$ & & & $48.0 \%$ & $1.1 \%$ & \\
\hline$v 1 h_{11 / 2} \rightarrow \pi 1 h_{9 / 2}$ & & & & $10.1 \%$ & $88.1 \%$ \\
\hline
\end{tabular}

that the inclusion of $T=1$ pairing increases the GT energies for transitions below $12 \mathrm{MeV}$. For the main peak of GTR, the inclusion of $T=1$ pairing results in the splitting of transition, and the centroid energy in the energy region $12 \sim 22 \mathrm{MeV}$ is also increased from 15.4 to $16.4 \mathrm{MeV}$. To understand this GT strength splitting, the main neutron-to-proton (Q)RPA amplitudes $\left(X_{p h}^{2}-Y_{p h}^{2}>1 \%\right)$ for different GT excitations in ${ }^{118} \mathrm{Sn}$ calculated without and with the $T=1$ pairing interaction are given in Table I. Due to the pairing correlation, the neutrons are scattered to higher levels in $N=50 \sim 82$ shell, and hence occupy the $h_{11 / 2}$ level. Therefore, a transition dominated by the new configuration $v 1 h_{11 / 2} \rightarrow \pi 1 h_{9 / 2}$ appears and meanwhile the transition at $E \approx 15 \mathrm{MeV}$ is mixed with new configurations from $v 1 h_{11 / 2}$. In addition, the transition at $E=9.9 \mathrm{MeV}$ is also mixed with a new configuration from $v 1 h_{11 / 2}$, whose QRPA amplitude even reaches $50 \%$.

In addition to the isovector $T=1$ pairing interaction, the isoscalar $T=0$ pairing interaction also plays an important role in describing the GTR [11,44]. Figure 11 shows the effects of $T=0$ pairing interaction on the GT strength distribution in ${ }^{118} \mathrm{Sn}$, where $V_{0}$ is the strength of the $T=0$ pairing interaction. Clearly, the excitation energy of the main peak is less affected by the $T=0$ pairing. However, the $T=0$ pairing interaction reduces the excitation energies and transition strengths in the energy region higher than the main peak, and hence reduces the splitting of GTR in the energy region $12 \sim 22 \mathrm{MeV}$. In the energy region lower than the main peak, the $T=0$ pairing interaction also reduces the excitation energies while it increases the transition strengths. From the QRPA amplitudes for the RHFB + QRPA calculations shown in Table I, it is known that the main peak at $14.9 \mathrm{MeV}$ is dominated by the configuration $\nu 1 g_{9 / 2} \rightarrow \pi 1 g_{7 / 2}$, which is almost a pure ph configuration with occupation probabilities $v^{2}\left(v 1 g_{9 / 2}\right)=0.99$ and $v^{2}\left(\pi 1 g_{7 / 2}\right)=0.00$. Therefore, the effect of $T=0$ pairing interaction on the main peak is relatively small. However, the peak at $18.3 \mathrm{MeV}$ is dominated 


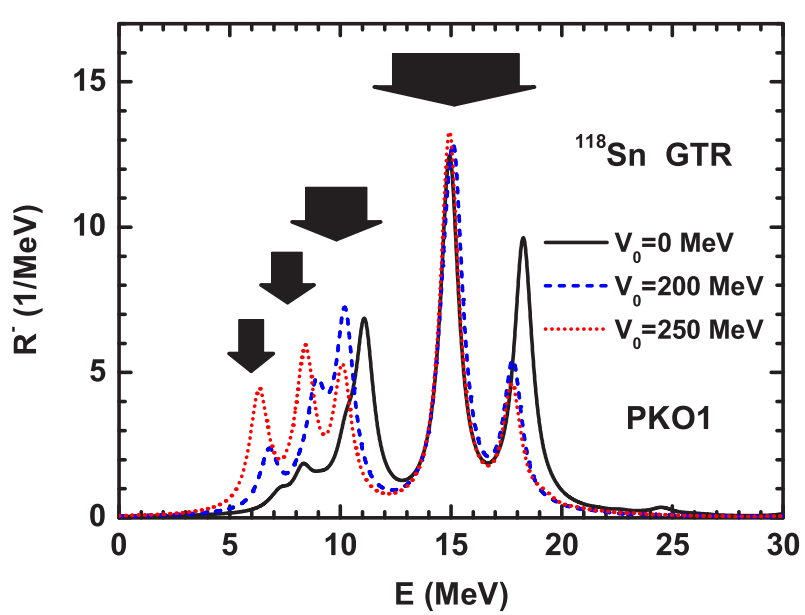

FIG. 11. GT strength distribution in ${ }^{118} \mathrm{Sn}$ calculated by the RHFB + QRPA approach with PKO1 for different values of $V_{0}$. The experimental data [90] are shown with arrows, whose widths illustrate the widths of the corresponding resonances.

by the configuration $v 1 h_{11 / 2} \rightarrow \pi 1 h_{9 / 2}$, which is more like a pp configuration with occupation probabilities $v^{2}\left(v 1 h_{11 / 2}\right)=$ 0.21 and $v^{2}\left(\pi 1 h_{9 / 2}\right)=0.00$, and thus the attractive $T=0$ pairing interaction reduces its excitation energy. For the peak at $11.1 \mathrm{MeV}$, its main configuration is $\nu 1 h_{11 / 2} \rightarrow \pi 1 h_{11 / 2}$, so the $T=0$ pairing interaction also has an important effect on this transition. For comparison, the main QRPA amplitudes $\left(X_{p h}^{2}-Y_{p h}^{2}>1 \%\right)$ for these three GT transitions calculated by including the $T=0$ pairing interaction with $V_{0}=250 \mathrm{MeV}$ are given in Table II. Clearly, the main QRPA amplitudes are remarkably affected by the $T=0$ pairing interaction, especially for those transitions dominated by the pp-type configurations.

For comparison, the experimental GT excitation energies and widths in ${ }^{118} \mathrm{Sn}$ are also shown in Fig. 11, which are named to be GT1, GT2, GT3, and GT4 as the decrease of their GT energies similar to Ref. [90]. The two peaks in the energy region $12 \sim 22 \mathrm{MeV}$ correspond to the GT1, while the predicted splitting of the GTR could not be observed, since the total width of the main resonance is of about $6 \mathrm{MeV}$ [90]

TABLE II. Main neutron-to-proton QRPA amplitudes $\left(X_{p h}^{2}-\right.$ $Y_{p h}^{2}>1 \%$ ) for different GT excitations in ${ }^{118} \mathrm{Sn}$ calculated by including the $T=0$ pairing interaction with $V_{0}=250 \mathrm{MeV}$. Excitation energies are in unit of $\mathrm{MeV}$.

\begin{tabular}{lclc}
\hline \hline Configurations & $E=10.1$ & 14.9 & 17.8 \\
\hline$\nu 1 g_{9 / 2} \rightarrow \pi 1 g_{7 / 2}$ & $2.7 \%$ & $89.6 \%$ & $4.6 \%$ \\
$\nu 1 g_{7 / 2} \rightarrow \pi 1 g_{7 / 2}$ & $2.8 \%$ & & \\
$\nu 1 g_{7 / 2} \rightarrow \pi 2 d_{5 / 2}$ & $1.9 \%$ & & \\
$\nu 2 d_{5 / 2} \rightarrow \pi 2 d_{5 / 2}$ & $3.7 \%$ & & \\
$\nu 2 d_{5 / 2} \rightarrow \pi 2 d_{3 / 2}$ & $81.9 \%$ & $1.6 \%$ & \\
$\nu 2 d_{5 / 2} \rightarrow \pi 3 d_{5 / 2}$ & & & $4.7 \%$ \\
$\nu 2 d_{5 / 2} \rightarrow \pi 3 d_{3 / 2}$ & & & $8.6 \%$ \\
$\nu 1 h_{11 / 2} \rightarrow \pi 1 h_{11 / 2}$ & $2.4 \%$ & & $4.5 \%$ \\
$\nu 1 h_{11 / 2} \rightarrow \pi 1 h_{9 / 2}$ & & $2.7 \%$ & $50.2 \%$ \\
$\nu 1 h_{9 / 2} \rightarrow \pi 1 h_{11 / 2}$ & & $3.2 \%$ & $29.7 \%$ \\
\hline \hline
\end{tabular}

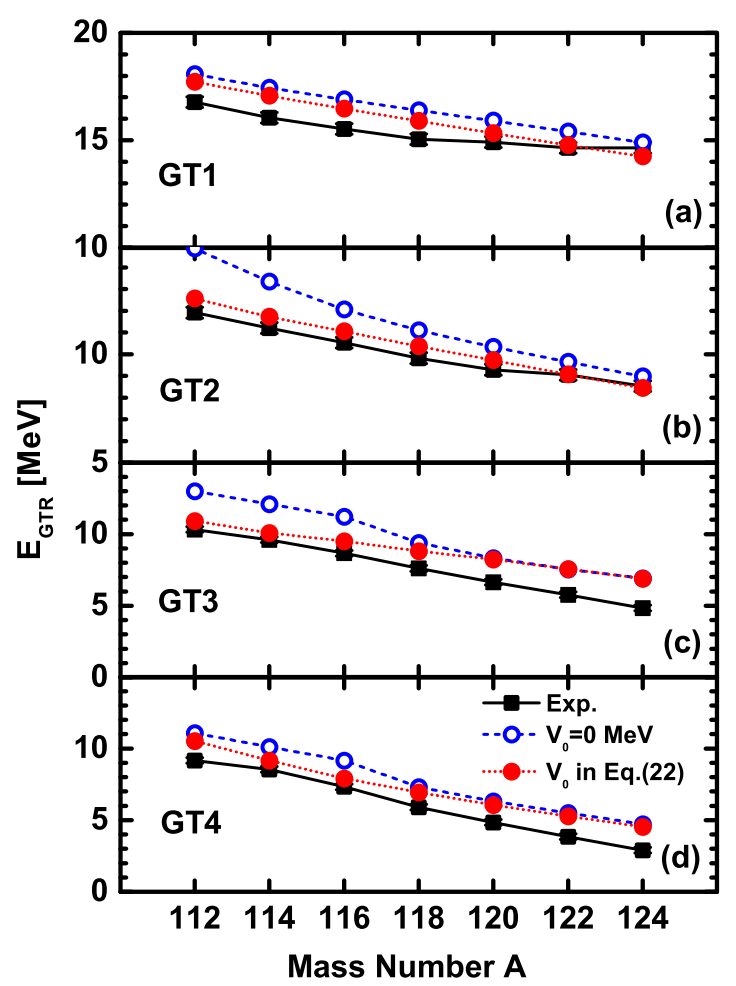

FIG. 12. GT excitation energies of the even-even $\mathrm{Sn}$ isotopes. The RHFB + QRPA calculations without and with the $T=0$ pairing in Eq. (22) are shown by the open and filled circles, respectively. The experimental values in Ref. [90] are denoted by the filled squares.

exceeding the predicted energy splitting. Clearly, the inclusion of $T=0$ pairing interaction improves the theoretical description of low-lying GT transitions. Then the GT2, GT3, and GT4 in ${ }^{118} \mathrm{Sn}$ are well predicted by the RHFB + QRPA approach.

The strength $V_{0}$ of $T=0$ pairing interaction is usually determined by fitting to the measured nuclear $\beta$-decay half-lives. A recent study based on the RHFB + QRPA approach found that an isospin-dependent $V_{0}$ can provide a good description of nuclear $\beta$-decay half-lives in the region of $20 \leqslant Z \leqslant 50$ [52]. With this isospin-dependent $V_{0}$ shown in Eq. (22), the calculated centroid energies for the GT1, GT2, GT3, and GT4 of the even-even Sn isotopes are shown in Fig. 12. Without the $T=0$ pairing interaction, the GT excitation energies are systematically higher than the experimental data. The $T=0$ pairing interaction can reduce the GT excitation energies and the agreements with the experimental data are improved systematically. In addition, it is found that the influence of $T=0$ pairing on the excitation energies of GT2, GT3, and GT4 decreases as the neutron number increases. This can be understood from the fact that the pairing effects become weaker and weaker when approaching the closed shell $N=82$.

\section{SUMMARY AND PERSPECTIVES}

In this work, the self-consistent quasiparticle random-phase approximation model is developed based on the relativistic Hatree-Fock-Bogoliubov theory, and it is then employed to 
study the nuclear isobaric analog states and Gamov-Teller resonances by taking the $\mathrm{Sn}$ isotopes as examples. It is found that the particle-particle residual interaction is essential to concentrate the IAS in a single peak for open-shell nuclei and the Coulomb exchange terms are very important to predict the IAS energies. For the GTR, the isoscalar $\sigma$ and $\omega$ mesons play an crucial role in the particle-hole residual interactions via the exchange terms. The isovector pairing can increase the calculated GTR energies and result in new excitations as the pairing scatters nucleons to higher energy levels. The isoscalar pairing has a strong influence on the low-lying tail of the GTR and is necessary to reproduce the experimental GTR energies.

The present RHFB + QRPA approach can also be employed to study other nuclear charge-exchange excitations, such as the spin-dipole and spin-quadrupole resonances. The predicted properties of charge-exchange excitations can be further used to calculate other nuclear weak-interaction processes beside nuclear $\beta$-decay half-lives, such as nuclear electron capture and neutrino-nucleus scattering. In addition, the present QRPA approach are formulated with the spherical symmetry, so it is worthwhile to extend the present approach by including deformation degree of freedom in the future for better describing the properties of deformed nuclei.

\section{ACKNOWLEDGMENTS}

This work was partly supported by the Major State 973 Program of China (Grant No. 2013CB834400), the National Natural Science Foundation of China (Grants No. 11205004, No. 11335002, No. 11305161, No. 11375076, No. 11675065 , and No. 11621131001), the Natural Science Foundation of Anhui Province under Grant No. 1708085QA10, the Key Research Foundation of Education Ministry of Anhui Province under Grant No. KJ2016A026, the Specialized Research Fund for the Doctoral Program of Higher Education under Grant No. 20130211110005, and the RIKEN iTHES and iTHEMS projects.
[1] I. Tanihata, H. Hamagaki, O. Hashimoto, Y. Shida, N. Yoshikawa, K. Sugimoto, O. Yamakawa, T. Kobayashi, and N. Takahashi, Phys. Rev. Lett. 55, 2676 (1985).

[2] J. Meng and P. Ring, Phys. Rev. Lett. 77, 3963 (1996).

[3] A. Ozawa, T. Kobayashi, T. Suzuki, K. Yoshida, and I. Tanihata, Phys. Rev. Lett. 84, 5493 (2000).

[4] D. Vretenar, A. V. Afanasjev, G. A. Lalazissis, and P. Ring, Phys. Rep. 409, 101 (2005).

[5] J. Meng, H. Toki, S. G. Zhou, S. Q. Zhang, W. H. Long, and L. S. Geng, Prog. Part. Nucl. Phys. 57, 470 (2006).

[6] T. Otsuka, T. Suzuki, M. Honma, Y. Utsuno, N. Tsunoda, K. Tsukiyama, and M. Hjorth-Jensen, Phys. Rev. Lett. 104, 012501 (2010).

[7] A. Krasznahorkay et al., Phys. Rev. Lett. 82, 3216 (1999).

[8] J. C. Hardy and I. S. Towner, Phys. Rev. C 91, 025501 (2015).

[9] H. Liang, N. Van Giai, and J. Meng, Phys. Rev. C 79, 064316 (2009).

[10] K. Langanke and G. Martínez-Pinedo, Rev. Mod. Phys. 75, 819 (2003).

[11] J. Engel, M. Bender, J. Dobaczewski, W. Nazarewicz, and R. Surman, Phys. Rev. C 60, 014302 (1999).

[12] N. Paar, D. Vretenar, T. Marketin, and P. Ring, Phys. Rev. C 77, 024608 (2008).

[13] Y. F. Niu, N. Paar, D. Vretenar, and J. Meng, Phys. Rev. C 83, 045807 (2011).

[14] F. Osterfeld, Rev. Mod. Phys. 64, 491 (1992).

[15] Y. Fujita, B. Rubio, and W. Gelletly, Prog. Part. Nucl. Phys. 66, 549 (2011).

[16] D. Frekers, P. Puppe, J. H. Thies, and H. Ejiri, Nucl. Phys. A 916, 219 (2013).

[17] E. Caurier, G. Martínez-Pinedo, F. Nowacki, A. Poves, and A. P. Zuker, Rev. Mod. Phys. 77, 427 (2005).

[18] G. Martínez-Pinedo and K. Langanke, Phys. Rev. Lett. 83, 4502 (1999).

[19] Q. Zhi, E. Caurier, J. J. Cuenca-García, K. Langanke, G. Martínez-Pinedo, and K. Sieja, Phys. Rev. C 87, 025803 (2013).

[20] J. Krumlinde and P. Möller, Nucl. Phys. A 417, 419 (1984).
[21] A. Staudt, E. Bender, K. Muto, and H. V. Klapdor-Kleingrothaus, At. Data Nucl. Data Tables 44, 79 (1990).

[22] M. Hirsch, A. Staudt, and H.-V. Klapdor-Kleingrothaus, At. Data Nucl. Data Tables 51, 243 (1992).

[23] P. Möller and J. Randrup, Nucl. Phys. A 514, 1 (1990).

[24] P. Möller, J. R. Nix, and K.-L. Kratz, At. Data Nucl. Data Tables 66, 131 (1997).

[25] A. Hektor, E. Kolbe, K. Langanke, and J. Toivanen, Phys. Rev. C 61, 055803 (2000).

[26] D. D. Ni and Z. Z. Ren, J. Phys. G 39, 125105 (2012).

[27] F. Krmpotić, K. Ebert, and W. Wild, Nucl. Phys. A 342, 497 (1980).

[28] N. Auerbach and A. Klein, Phys. Lett. B 106, 347 (1981).

[29] N. Auerbach and A. Klein, Phys. Rev. C 30, 1032 (1984).

[30] P. Sarriguren and J. Pereira, Phys. Rev. C 81, 064314 (2010).

[31] P. Sarriguren, A. Algora, and J. Pereira, Phys. Rev. C 89, 034311 (2014).

[32] I. N. Borzov, S. A. Fayans, E. Krömer, and D. Zawischa, Z. Phys. A 355, 117 (1996).

[33] I. N. Borzov and S. Goriely, Phys. Rev. C 62, 035501 (2000).

[34] S. Fracasso and G. Colò, Phys. Rev. C 72, 064310 (2005).

[35] S. Fracasso and G. Colò, Phys. Rev. C 76, 044307 (2007).

[36] K. Yoshida, Prog. Theor. Exp. Phys. 2013, 113D02 (2013).

[37] M. T. Mustonen, T. Shafer, Z. Zenginerler, and J. Engel, Phys. Rev. C 90, 024308 (2014).

[38] M. T. Mustonen and J. Engel, Phys. Rev. C 93, 014304 (2016).

[39] T. Nakatsukasa, T. Inakura, and K. Yabana, Phys. Rev. C 76, 024318 (2007).

[40] H. Z. Liang, T. Nakatsukasa, Z. M. Niu, and J. Meng, Phys. Rev. C 87, 054310 (2013).

[41] C. L. Bai, H. Q. Zhang, H. Sagawa, X. Z. Zhang, G. Colò, and F. R. Xu, Phys. Rev. Lett. 105, 072501 (2010).

[42] F. Minato and C. L. Bai, Phys. Rev. Lett. 110, 122501 (2013).

[43] L. J. Jiang, S. Yang, B. Y. Sun, W. H. Long, and H. Q. Gu, Phys. Rev. C 91, 034326 (2015).

[44] N. Paar, T. Nikšić, D. Vretenar, and P. Ring, Phys. Rev. C 69, 054303 (2004). 
[45] T. Nikšić, T. Marketin, D. Vretenar, N. Paar, and P. Ring, Phys. Rev. C 71, 014308 (2005).

[46] P. Ring, Prog. Part. Nucl. Phys. 37, 193 (1996).

[47] H. Z. Liang, J. Meng, and S. G. Zhou, Phys. Rep. 570, 1 (2015).

[48] J. Meng and S. G. Zhou, J. Phys. G 42, 093101 (2015).

[49] International Review of Nuclear Physics: Vol. 10, Relativistic Density Functional for Nuclear Structure, edited by J. Meng (World Scientific, Singapore, 2016).

[50] B. Sun, F. Montes, L. S. Geng, H. Geissel, Yu. A. Litvinov, and J. Meng, Phys. Rev. C 78, 025806 (2008).

[51] Z. M. Niu, B. Sun, and J. Meng, Phys. Rev. C 80, 065806 (2009).

[52] Z. M. Niu, Y. F. Niu, H. Z. Liang, W. H. Long, T. Nikšić, D. Vretenar, and J. Meng, Phys. Lett. B 723, 172 (2013).

[53] C. De Conti, A. P. Galeão, and F. Krmpotić, Phys. Lett. B 444, 14 (1998).

[54] P. Ring, Z. Y. Ma, N. Van Giai, D. Vretenar, A. Wandelt, and L. G. Cao, Nucl. Phys. A 694, 249 (2001).

[55] Z. Y. Ma, B. Q. Chen, N. Van Giai, and T. Suzuki, Eur. Phys. J. A 20, 429 (2004).

[56] N. Paar, P. Ring, T. Nikšić, and D. Vretenar, Phys. Rev. C 67, 034312 (2003).

[57] P. Finelli, N. Kaiser, D. Vretenar, and W. Weise, Nucl. Phys. A 791, 57 (2007).

[58] T. Marketin, D. Vretenar, and P. Ring, Phys. Rev. C 75, 024304 (2007).

[59] Z. Y. Wang, Y. F. Niu, Z. M. Niu, and J. Y. Guo, J. Phys. G 43, 045108 (2016).

[60] Z. M. Niu, Y. F. Niu, Q. Liu, H. Z. Liang, and J. Y. Guo, Phys. Rev. C 87, 051303(R) (2013).

[61] T. Marketin, L. Huther, and G. Martínez-Pinedo, Phys. Rev. C 93, 025805 (2016).

[62] W. H. Long, N. Van Giai, and J. Meng, Phys. Lett. B 640, 150 (2006).

[63] W. H. Long, H. Sagawa, N. V. Giai, and J. Meng, Phys. Rev. C 76, 034314 (2007).

[64] H. Z. Liang, N. Van Giai, and J. Meng, Phys. Rev. Lett. 101, 122502 (2008).

[65] H. Z. Liang, P. W. Zhao, and J. Meng, Phys. Rev. C 85, 064302 (2012).

[66] W. H. Long, H. Sagawa, J. Meng, and N. Van Giai, Europhys. Lett. 82, 12001 (2008).

[67] W. H. Long, T. Nakatsukasa, H. Sagawa, J. Meng, H. Nakada, and Y. Zhang, Phys. Lett. B 680, 428 (2009).
[68] J. Dobaczewski, H. Flocard, and J. Treiner, Nucl. Phys. A 422, 103 (1984).

[69] J. Meng, Nucl. Phys. A 635, 3 (1998).

[70] W. H. Long, P. Ring, N. Van Giai, and J. Meng, Phys. Rev. C 81, 024308 (2010).

[71] W. H. Long, P. Ring, J. Meng, N. Van Giai, and Carlos A. Bertulani, Phys. Rev. C 81, 031302(R) (2010).

[72] J. J. Li, J. Margueron, W. H. Long, and N. Van Giai, Phys. Rev. C 92, 014302 (2015).

[73] J. J. Li, J. Margueron, W. H. Long, and N. Van Giai, Phys. Lett. B 753, 97 (2016).

[74] J. J. Li, W. H. Long, J. Margueron, and N. Van Giai, Phys. Lett. B 732, 169 (2014).

[75] L. P. Gor'kov, Sov. Phys. JETP 7, 505 (1958).

[76] P. Ring and P. Schuck, The Nuclear Many-Body Problem (Springer-Verlag, Heidelberg, 1980).

[77] H. Kucharek and P. Ring, Z. Phys. A: Hadrons Nucl. 339, 23 (1991)

[78] J. F. Berger, M. Girod, and D. Gogny, Nucl. Phys. A 428, 23 (1984).

[79] S. G. Zhou, J. Meng, and P. Ring, Phys. Rev. C 68, 034323 (2003).

[80] G. Audi, F. G. Kondev, M. Wang, B. Pfeiffer, X. Sun, J. Blachot, and M. MacCormick, Chin. Phys. C 36, 1157 (2012).

[81] G. A. Lalazissis, T. Nikšić, D. Vretenar, and P. Ring, Phys. Rev. C 71, 024312 (2005).

[82] M. Wang, G. Audi, A. H. Wapstra, F. G. Kondev, M. MacCormick, X. Xu, and B. Pfeiffer, Chin. Phys. C 36, 1603 (2012).

[83] A. Trzcinska, J. Jastrzebski, P. Lubinski, F. J. Hartmann, R. Schmidt, T. von Egidy, and B. Klos, Phys. Rev. Lett. 87, 082501 (2001).

[84] S. Terashima et al., Phys. Rev. C 77, 024317 (2008).

[85] L. W. Chen, C. M. Ko, and B. A. Li, Phys. Rev. C 72, 064309 (2005).

[86] L. W. Chen, Nucl. Phys. Rev. 31, 273 (2014).

[87] C. A. Engelbrecht and R. H. Lemmer, Phys. Rev. Lett. 24, 607 (1970).

[88] K. Ikeda, S. Fujii, and J. I. Fujita, Phys. Lett. 3, 271 (1963).

[89] H. Kurasawa, T. Suzuki, and N. Van Giai, Phys. Rev. Lett. 91, 062501 (2003).

[90] K. Pham, J. Janecke, D. A. Roberts, M. N. Harakeh, G. P. A. Berg, S. Chang, J. Liu, E. J. Stephenson, B. F. Davis, H. Akimune, and M. Fujiwara, Phys. Rev. C 51, 526 (1995). 